\section{Waste disposal under the spotlight}

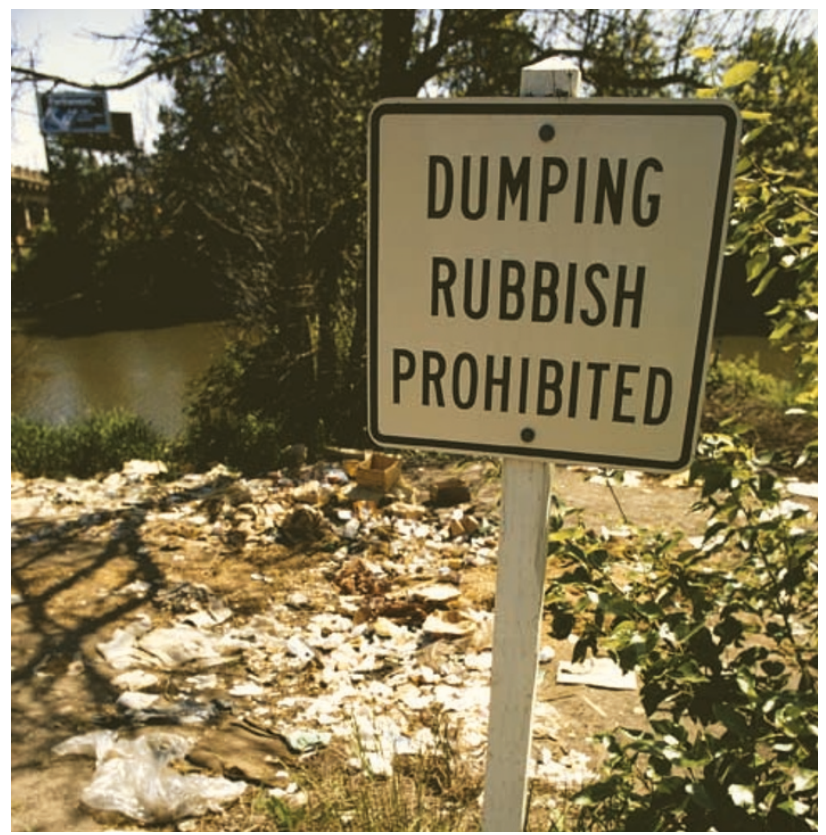

Destruction of key regulatory proteins by the ubiquitin/proteasome system is crucial for many regulatory processes in the cell, and the system has been proposed to have a role in a number of disorders, including cancer and neurodegenerative diseases. Proteasome inhibitors, such as bortezomib, are promising drugs for cancer treatment as they have a profound antitumour activity. On the other hand, it has been suggested that impairment of the system might play a role in neuronal cell death in various neurodegenerative disorders. Despite the existence of animal models for a number of these diseases, such as Alzheimer's and Parkinson's disease, in vivo data concerning the ubiquitin/proteasome system are lacking. To address this need, Dantuma and colleagues have developed a model for in vivo quantitative analysis of the degradative machinery by generating transgenic mice carrying a green fluorescent protein (GFP) reporter with a constitutively active degradation signal, according to work published in Nature Biotechnology.

The process of removing intracellular proteins involves a complex network of enzymes that link multiple N-terminally linked ubiquitin molecules to the protein substrate, which targets the substrate for unfolding and subsequent degradation by the proteasome. In order to dissect this process in vivo, Lindsten et al. selected a GFP-reporter fused to an $\mathrm{N}$-terminally linked ubiquitin that serves as an acceptor for further ubiquitin molecules, thereby automatically delivering the GFP to the proteasome for degradation. The GFP fusion protein was tolerated at high concentration in mammalian cells. Proteasome inhibitors given to the transgenic animals expressing this reporter substrate resulted in substantial accumulation of GFP in multiple tissues,

\section{Seeing the forest for the trees}

The path from molecular genetics and biology to treatments is proving to be a long one, with incremental steps occurring, rather than the initially predicted large leaps. Most advances have been made in the field of toxicogenomics, as characteristic transcriptional responses to cellular insults have allowed the generation of drug-toxicity expression profiles. What has not been investigated as thoroughly is whether treatments administered at non-toxic doses also produce distinct expression profiles that correlate with clinical efficacy.

To examine this, Gunther and colleagues at CuraGen looked at the transcriptional responses to several antidepressants, antipsychotics and opioid-receptor agonists in human primary neurons. In Proceedings of the National Academy of Sciences, they report that the classification tree (CT) and random forest (RF) methods of analysis which classify patterns of novel data on the basis of prior knowledge of sample classes - can accurately predict the physiological consequences of treatments from their gene expression profiles.

Thirty-six treatments were tested, including the tricyclic, selective serotoninreuptake inhibitors (SSRIs), monoamine oxidase inhibitors and atypical subclasses of antidepressants, classic and atypical antipsychotics, and $\delta$-, $\kappa$ - and $\mu$-opioidreceptor agonists. Of these, the $\mathrm{CT}$ method classified 32 out of 36 expression profiles correctly as being antidepressants, antipsychotics or opioid-receptor agonists, whereas the RF method classified 30 out of 36 . This difference in accuracy is probably caused by relative emphases on marker strength - the CT method identifies a few genes that each explain a large portion of the differences among classes, whereas the RT method identifies many genes, each of which accounts for small differences. Repeating the construction of the models with the omission of expression data from an entire subclass could still predict the functional class of the uncharacterized drugs. All five SSRIs and ten tricyclics were correctly predicted as being antidepressants in an 'SSRI-less' model and a 'tricyclic-less' model, respectively, even though neither model was constructed with representative examples of each subclass.
The expansion of the assay to other drug classes will require the refinement of false positives, and will be limited to the number of classes in which a given cell type is responsive. Nevertheless, this represents an important step forward in the screening of novel molecules, as previously the activity of such molecules could only be determined in animal studies or clinical trials. The finding that subclasses that are mechanistically heterogeneous show similar expression profiles indicates that they share downstream mechanisms, and has several potential applications. For example, this could be important in the validation of genes and the prioritization of lead compounds early in drug development. And the finding that the method can classify functionality, whether or not the subclass is known, could persuade drug developers not to go down the 'me too' route, and instead seek novel therapeutically viable compounds.

Simon Frantz

(i) References and links ORIGINAL RESEARCH PAPER Gunther, E. C., Stone, D. J., Gerwien, R. W., Bento, P. \& Heyes, M. P. Prediction of clinical drug efficacy by classification of drug-induced genomic expression profiles in vitro. Proc. Natt Acad. Sci. USA 100 9608-9613 (2003)

FURTHER READING Lindpaintner, K. Science and society: The impact of pharmacogenetics and pharmacogenomics on drug discovery. Nature Rev. Drug Discov. 1, 463-469 (2002) 
confirming the in vivo functionality of the reporter. Furthermore, analysis of the transgenic animals revealed that accumulation of the reporter was induced in primary neurons by an aberrant ubiquitin found in Alzheimer's disease.

The role of the ubiquitin/proteasome sytem in diverse disorders, as well as its identification as a therapeutic target, makes these GFP-transgenic animals an important tool for monitoring the status of the ubiquitin/ proteasome system in physiological or pathological conditions.

Melanie Brazil

(i) References and links ORIGINAL RESEARCH PAPER Lindsten, K. etal. A transgenic mouse model of the ubiquitin/ proteasome system. Nature Biotechnol. 2003 July 20 (doi:10.1038/nbt851) FURTHER READING Berke, S. J. \& Paulson, H. L . Protein aggregation and the ubiquitin proteasome pathway: gaining the UPPer hand on neurodegeneration. Curr. Opin. Genet. Dev. 13, 253-261 (2003) | Jesenberger, V. \& Jentsch, S. Deadly encounter: ubiquitin meets apoptosis. Nature Rev. Mol. Cell Biol. 3, 112-121 (2002) | Adams, J. Proteasome inhibitors as new anticancer drugs. Curr. Opin. Oncol. 14 628-634 (2002)

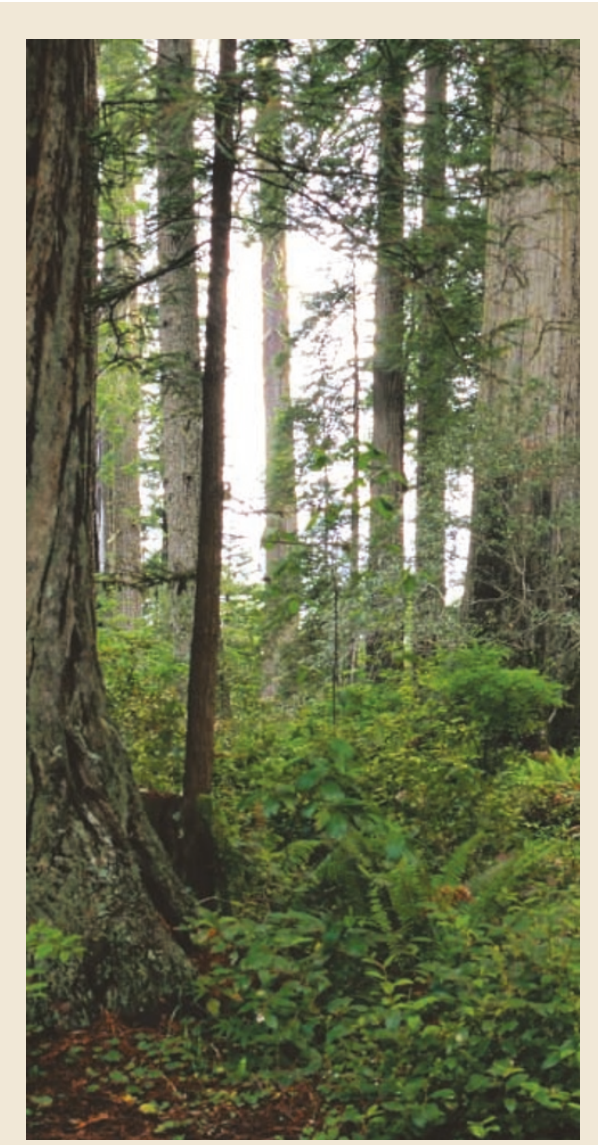

\section{VIRTUAL SCREENING}

\section{Treasure-hunting tips}

Although high-throughput screening (HTS) is firmly established as a valuable method for identifying hits from compound collections, it is widely recognized that the quantity, quality and diversity of the hits identified has been below original expectations. A recent paper in the Journal of Medicinal Chemistry describes a strategy that could increase the chances of screening campaigns striking gold.

The approach put forward by Mestres and Veeneman aims to address the intrinsic incompleteness of screening libraries. Such libraries typically contain $\sim 10^{6}$ compounds, a number that is dwarfed by the potential number of synthesizable drug-like compounds, which has been estimated to be $>10^{18}$. Given this disparity, it seems unlikely that compounds possessing the optimal structural features, arranged in an optimal way around a core structure to bind to a particular protein target, will commonly be present in a screening collection.

However, as Mestres and Veeneman note, the probability that compounds are present that have almost the right structural features, arranged almost optimally to bind to a target protein (which, therefore, might be easily converted to high-quality hits), might be considerably greater. Such 'latent hits' could well remain undetected in standard HTS campaigns, but might be identifiable with the help of some knowledge of the key structural features for binding — for example, from known ligands of the target protein.

To test the idea that latent hits are present in screening collections and can be identified and easily 'promoted' to hits, the authors set out to find nonsteroidal agonists of the oestrogen receptor- $\alpha(E R-\alpha)$. First, a screening collection containing 133,836 compounds was filtered to remove compounds with a low probability of interacting with ER- $\alpha$ using information on key structural features of ER- $\alpha$ agonists. The resulting set of 11,047 compounds was then virtually screened using a ligand-based flexible superposition approach based on the natural ER$\alpha$ agonist diethylstilbestrol (DES). Analysis of the top-ranking compounds from this screen identified two potential latent hits - compounds that lacked only one or two of the key binding features of DES - that had insufficient activity $\left(\mathrm{EC}_{50}>10 \mu \mathrm{m}\right)$ to be identified as hits in a conventional screen.

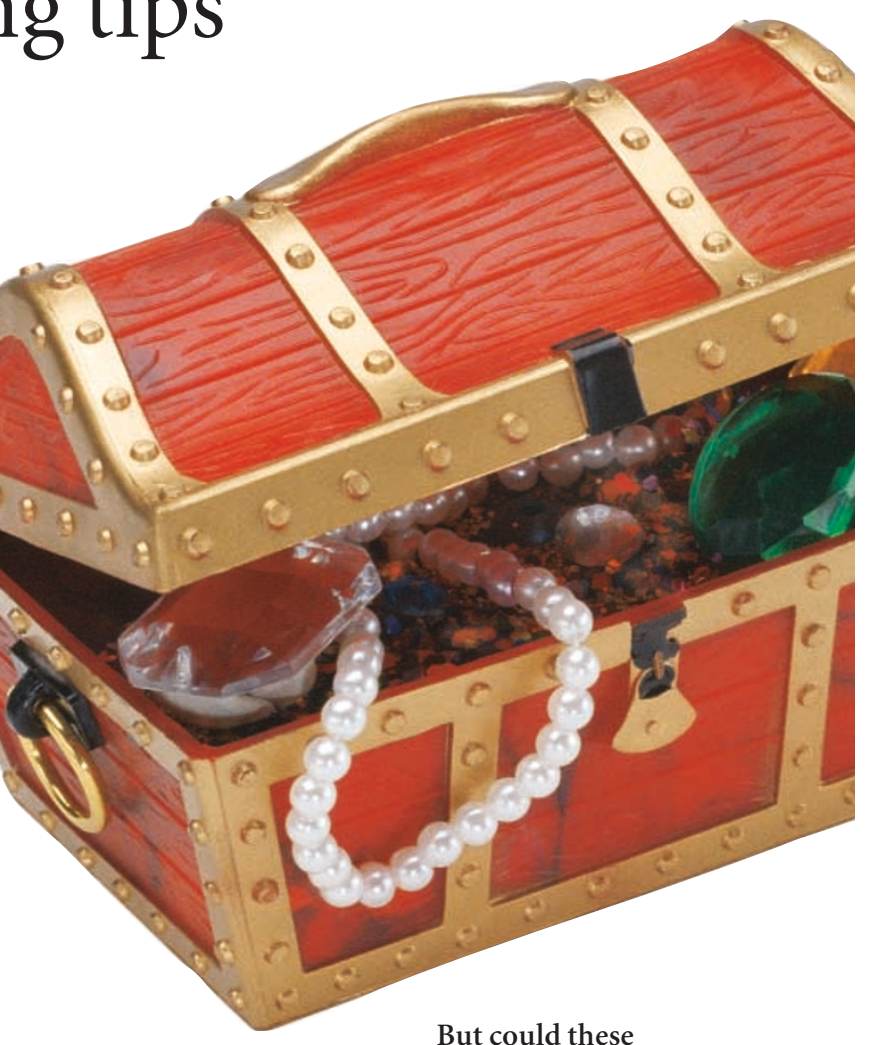

latent hits be promoted?

Inspection of the predicted binding mode of the compounds indicated simple structural modifications that could be made to fully mirror the key binding features of DES. Indeed, when these modified compounds were synthesized and tested in vitro, one had an $\mathrm{EC}_{50}$ of $0.004 \mu \mathrm{m}$, and the other an $\mathrm{EC}_{50}$ of $0.8 \mu \mathrm{m}$ - that is, both could be considered genuine hits - providing strong support for Mestres and Veeneman's approach. Furthermore, recognizing that all compounds belonging to a chemical series represented by an active compound for a particular target can be considered latent hits for other family-related targets is equivalent to recognizing that the scaffold contained in that active compound has the potential of being a 'privileged scaffold' for the entire target family. The authors' results therefore also provide support for current screening strategies based on targeted libraries designed around scaffolds present in active compounds.

Peter Kirkpatrick

(Q) References and links

ORIGINAL RESEARCH PAPER Mestres, J. \& Veeneman, G. H. Identification of "latent hits" in compound screening collections. J. Med. Chem. 46, 3441-3444 (2003)

FURTHER READING Bajorath, J. Integration of virtual and high-throughput screening. Nature Rev. Drug Discov. 1, 882-894 (2002) | Bleicher, K. H. et al. Hit and lead generation: beyond high-throughput screening. Nature Rev. Drug Discov. 2, 369-378 (2003) 